\title{
El papel de las personalidades de radio como prescriptores de complementos alimenticios. Un análisis de las declaraciones de propiedades saludables (H-RCs) en las menciones de radio en España
}

\author{
The Role of Radio Personalities as Endorsers of Food \\ Supplements. An Analysis of Health-Related Claims (H-RCs) on \\ Spanish Radio Mentions
}

Ana García-Arranz ${ }^{1}$. Universidad Rey Juan Carlos. España.

anamaria.garcia.arranz@urjc.es

$[\mathrm{CV}]$ (D) $\mathrm{G}$

Salvador Perelló-Oliver. Universidad Rey Juan Carlos. España.

salvador.perello@urjc.es

$[\mathrm{CV}] \odot \mathrm{G}$

Clara Muela-Molina. Universidad Rey Juan Carlos. España.

clara.muela@urjc.es

$[\mathrm{CV}] \odot \mathrm{G} \mathrm{R}^{\mathrm{E}} \mathrm{O}$

Este trabajo fue apoyado por el Ministerio de Economía y Competitividad del Gobierno de España, Programa Estatal de I+D Orientada a los Retos de la Sociedad, bajo la subvención CSO201782267-R. Fecha de inicio: 1 de enero de 2018. Fecha de finalización: 31 de diciembre de 2020

Cómo citar este artículo / Referencia normalizada

García-Arranz, A., Perelló-Oliver, S. \& Muela-Molina, C. (2021). El papel de las personalidades de radio como prescriptores de complementos alimenticios. Un análisis de las declaraciones de propiedades saludables (H-RCs) en las menciones de radio en España. Revista Latina de Comunicación Social, 79, 263-282. https://www.doi.org/10.4185/RLCS-2021-1511

\section{RESUMEN}

Introducción: Los anunciantes utilizan testimoniales debido a su potencial en términos de persuasión y eficacia del mensaje. El objetivo de este trabajo es analizar las declaraciones de propiedades saludables en la publicidad de complementos alimenticios estableciendo la relación entre las personalidades de radio y la información relacionada con la salud. Metodología: Se realizó

${ }^{1}$ Corresponding author. 
un análisis de contenido de todas las menciones radiales - con un corpus final de 437- emitidas en 2017 en las tres emisoras de radio generalista con mayor audiencia. Resultados: Predominan los locutores de radio (55\%) sobre los colaboradores (45\%), siendo las declaraciones funcionales el tipo más prevalente $(99,5 \%)$, seguido de las declaraciones de enfermedad, prohibidas por ley. Los colaboradores mencionan reclamos no autorizados con mayor frecuencia que los locutores. En relación a la información obligatoria sobre el contenido del producto, el $79 \%$ de las menciones no refiere ningún mineral que sustente sus supuestos beneficios. Discusión: Los anunciantes explotan la credibilidad de los líderes de opinión para respaldar los complementos alimenticios. Estas figuras son claramente inadecuadas para recomendar estos productos, ya que pueden implicar un gran riesgo para la salud de los consumidores. El uso significativo e ilícito de estas declaraciones exige una legislación más restrictiva y mecanismos de control más estrictos por parte de las autoridades autorreguladoras. Conclusiones: Si bien los anunciantes son los únicos responsables del anuncio, la responsabilidad debe extenderse a los responsables de los medios y personalidades de la radio, debido a su influencia social, para una mayor protección contra la publicidad ilícita.

PALABRAS CLAVE: publicidad; declaraciones de propiedades saludables; información de producto; complementos alimenticios; radio; personalidades de radio; credibilidad de la fuente.

\begin{abstract}
Introduction: Advertisers use endorsers due to their potential in terms of persuasion and message effectiveness. The objective of this work is to analyze health-related claims in food supplements advertising establishing the relationship between radio personalities and health-related information. Methodology: A content analysis of all radio mentions - with a final corpus of 437- broadcast in 2017 on the three news/talk radio stations with the largest audiences was conducted. Results: Radio hosts $(55 \%)$ predominate over collaborators (45\%), with function claims as the most prevalent type of claim (99.5\%), followed by disease claims, prohibited by law. Collaborators mention nonauthorized claims more frequently than radio hosts. Regarding mandatory information on product content, $79 \%$ of mentions do not refer to any mineral that supports their alleged benefits. Discussion: Advertisers exploit the reliability of opinion leaders to endorse food supplements. These personalities are clearly inadequate to recommend these products, which implies a great risk to consumers' health. The significant and illicit use of these claims demands more restrictive legislation and stricter control mechanisms by self-regulatory authorities. Conclusions: Although advertisers are solely responsible for the illicit endorsement, the responsibility must be extended to media owners and radio personalities, due to their social influence, for a greater protection against illicit advertising.
\end{abstract}

KEYWORDS: advertising; health claims; product information; food supplements; radio; media personality; source credibility.

\title{
CONTENIDO
}

1. Introducción. 1.1. La radio como medio publicitario. 1.2. Patrocinadores en la publicidad radiofónica. 2. Marco teórico y revisión de la literatura. 2.1. Patrocinadores en la publicidad de complementos alimenticios. 2.2. Declaraciones de propiedades saludables en complementos alimenticios. 3. Metodología. 4. Resultados. 5. Discusión. 5.1. Implicaciones de gestión. 5.2. Limitaciones y futura investigación. 6. Conclusiones. 7. Bibliografía.

Traducido por Paula González (Universidad Católica Andrés Bello, Venezuela) 


\section{Introducción}

\subsection{La radio como medio publicitario}

Pese a la creciente desconfianza en los medios de comunicación, la radio sigue siendo considerada el medio más fiable por el $42 \%$ de los españoles - porcentaje 8 y 11 puntos superior al de la prensa y televisión, respectivamente, y muy por encima del 14\% obtenido por las redes sociales - y el 58\% de los europeos (Comisión Europea, 2021). Esta confianza se traduce en 22.290.000 oyentes diarios (AIMC, 2021), de los cuales el 53,4\% elige emisoras de radio de noticias/entrevistas para estar al día de las últimas novedades. La programación de estas estaciones se basa en temas de actualidad de diversa índole, transmitidos a lo largo del día en diversos formatos. En este escenario informativo, los periodistas se convierten en el centro de atención, líderes de opinión que transmiten contenidos, así como la línea editorial de los propios programas.

La radio depende de la publicidad como su principal, y, a veces, exclusiva, fuente de financiamiento, con anuncios integrados en la programación como un elemento adicional del flujo de noticias, entre programas o secciones o, incluso dentro de ellos. De hecho, la tasa de ocupación publicitaria promedio en las estaciones de radio de noticias/entrevistas (N/TRS) es del 11\%, principalmente condensada por la mañana (AEA, 2021). En este sentido, la práctica más común es transmitir anuncios de radio en cortes comerciales — slots separados en la programación — de alrededor de 3 minutos (AEA, 2021), de modo que los oyentes puedan identificar los mensajes publicitarios del resto de la información, lo que genera una actitud relativamente desfavorable hacia estos cortes comerciales (Dens et al., 2018). Los anunciantes son conscientes de esta reacción y, por lo tanto, optan cada vez más por menciones en lugar de anuncios de radio (Espinosa Mirabet y Vico Blanco, 2016).

La práctica de integrar la publicidad en el contenido editorial es cada vez más común, y los periodistas responsables del programa o sus colaboradores suelen dar voz a las menciones. Esta inclusión de mensajes persuasivos en el contenido editorial puede afectar su procesamiento y dar lugar a diferencias en la evaluación del mensaje o en el recuerdo de la marca, debido a la credibilidad que los oyentes otorgan a la fuente. De esta forma, los mensajes comerciales se perciben de forma menos negativa y es menos probable que se eviten (van Reijmersdal, 2011). Esto es lo que se conoce como "transferencia de atención" (Moorman, et al., 2012; Dens et al., 2018; Stipp, 2018) de noticias a mensajes publicitarios.

A diferencia de otros formatos, como los programas musicales cuyo objetivo principal es el entretenimiento, la radio informativa requiere un mayor esfuerzo cognitivo y la implicación de los oyentes para prestar atención, entender y procesar la información para formarse una opinión. Los anunciantes aprovechan esta escucha activa para transmitir publicidad porque "la participación inducida por el programa contribuye a que se preste más atención a los anuncios que aparecen durante el programa" (Moorman et al., 2012, p. 27). Así, los anunciantes utilizan estos programas de información como una herramienta para mejorar la eficiencia publicitaria. Sin embargo, esta práctica viola y desdibuja el principio ético y tradicional que establece desde hace más de un siglo que las operaciones editoriales y comerciales deben separarse, incluso intentando legitimar su uso. De esta forma, a medida que aumenta el retorno potencial de la publicidad, disminuye la capacidad de los oyentes para activar voluntariamente sus filtros de atención ante los contenidos intrusivos o el discurso del periodista, que en realidad son contrarios al contexto informativo en el que se ubican y a la función objetivamente percibida del formato. Según Ha (2018), "al ocultar la finalidad publicitaria del contenido, se encuentra con la cuestión ética del engaño” (p. 153). 


\subsection{Patrocinadores en la publicidad radiofónica}

La publicidad tiene un papel central para la industria de los complementos alimenticios, con comerciales que, directa o indirectamente, promueven su consumo como una alternativa para los medicamentos (Royne et al., 2016) mientras promueven el autocuidado en lugar de la atención de los profesionales de la salud. En consecuencia, la mayoría de los países han promulgado medidas reglamentarias destinadas a controlar las actividades comerciales de los suplementos alimenticios y las declaraciones de propiedades saludables (H-RCs) anunciadas.

Emplear celebridades, consumidores típicos, expertos profesionales, empleados de la empresa o voceros anónimos como prescriptores publicitarios es una estrategia recurrente entre los anunciantes (Perelló-Oliver et al., 2018; Schouten et al., 2020; Tran et al., 2019), ya que imbuyen de personalidad a las marcas y maximizan el impacto persuasivo del mensaje. El objetivo es crear una imagen de marca a través de la persona y no del producto en sí, con el fin de capitalizar la confianza percibida de los consumidores potenciales (Mospan \& Alexander, 2018). Las personalidades de la radio pueden entenderse como celebridades prescriptoras, definidas como individuos que tienen reconocimiento público y que alinean su imagen con un producto o marca (McCracken, 1989). Conscientes de su mayor efectividad (Morimoto, 2020; Schouten et al., 2020), los anunciantes buscan incrementar el retorno de su inversión apalancando el impacto de este tipo de prescriptores en la audiencia en términos de credibilidad, influencia y confianza.

Además, los anuncios integrados en los programas de noticias son más efectivos y memorables, ya que los oyentes los perciben como más interesantes y, por lo tanto, mantienen su atención por más tiempo. En este sentido, muchos profesionales del campo publicitario favorecen el uso de periodistas como voceros de marca, y de menciones, es decir, anuncios leídos en vivo dentro del programa por los presentadores, equipo o compañeros de trabajo. Sin embargo, los detractores argumentan que la dilución de los límites entre contenidos editoriales y publicitarios (Muela-Molina et al., 2020) convierte a estos últimos en un pretexto para influir en la decisión de compra de los oyentes violando el principio de independencia periodística. Además, cabe señalar que el Punto 6 del código deontológico del periodista español — su cumplimiento, aunque no legalmente exigido, es obligatorio para los miembros de la profesión, que pueden ser expulsados de la asociación, aunque no inhabilitados para el ejercicio de la profesión, en caso de vulneración de algún principio recogido en el código- establece que, para evitar engaños o confusión en los usuarios, los periodistas deben hacer una distinción formal y rigurosa entre información y publicidad, y el Punto 7 afirma que los periodistas no deben aceptar, directamente o indirectamente, pagos o recompensas de terceros para promover, orientar, influir o publicar información u opiniones de cualquier naturaleza (FAPE, 2017). Estos principios deontológicos sientan las bases y razones de este trabajo, especialmente si se considera que los líderes de opinión y sus colaboradores cooperan con los anunciantes para promover los supuestos beneficios para la salud de productos cuyo consumo puede conllevar riesgos para la salud.

Aunque estudios previos han analizado las características de las menciones radiofónicas en general (Perelló-Oliver \& Muela-Molina, 2017) y la presencia de los diferentes tipos de prescriptores en los anuncios radiofónicos de suplementos dietéticos (Muela-Molina et al., 2021) en el contexto europeo, los estudios en este campo de investigación centrados en la información de productos relacionados con la salud en la publicidad de complementos alimenticios son escasos. Baudischova et al. (2018) se han centrado en la calidad de la información de Internet sobre los complementos alimenticios más vendidos en la República Checa, analizando sus sustancias, composición general y la legalidad de las declaraciones de propiedades saludables. Sin embargo, no se ha encontrado ningún trabajo sobre el 
análisis del papel de las personalidades de la radio que respaldan los complementos alimenticios desde la perspectiva del marco regulatorio europeo de los H-RCs.

El presente trabajo contribuye al campo al establecer la correlación entre las personalidades de la radio como fuentes confiables y la información del producto relacionada con la salud. El objetivo de este trabajo es analizar las declaraciones de propiedades saludables en la publicidad de complementos alimenticios en las emisoras de radio de noticias/entrevistas en España, estableciendo la relación entre las personalidades de la radio y la información relacionada con la salud.

\section{Marco teórico y revisión de la literatura}

\subsection{Patrocinadores en la publicidad de complementos alimenticios}

Los prescriptores se consideran una fuente de credibilidad. McCroskey y Young (1981) indicaron que la credibilidad es una actitud hacia la fuente de información, que modifica la aceptación del mensaje (Schouten et al., 2020; Tran et al., 2019) y el grado de acuerdo con el mismo. Por tanto, en la medida en que los mensajes no puedan recibirse fuera de su fuente, la credibilidad es un factor decisivo en la eficacia de la persuasión (Morimoto, 2020; Plasek \& Temesi, 2019). De hecho, la trascendencia de la credibilidad de la fuente en el proceso de comunicación es tal que se vuelve vital en la elección del vocero radial (Martín-Santana et al., 2017). McGinnies y Ward (1980) introdujeron la experiencia del comunicador confiable como un medio para fomentar la persuasión. De esta forma, dado que la confiabilidad está relacionada con la honestidad percibida de la fuente, la experiencia está relacionada con su conocimiento y competencia. La suposición de los consumidores de que una fuente experta proporcionará información precisa reduce su sensación de incertidumbre, por lo que evitan la tarea de examinar el mensaje, que aceptan ciegamente como válido (Teeny et al., 2017).

Investigaciones anteriores sobre anuncios de suplementos dietéticos han destacado a las celebridades como la principal fuente de patrocinio (Ethan et al., 2016). Sin embargo, no ha sido posible encontrar investigaciones sobre el uso de personalidades de los medios en la publicidad de complementos alimenticios. En consecuencia, y en este contexto, la primera pregunta de investigación se propone de la siguiente manera:

PI $\mathbf{I}_{1}$ ¿Cuál es la presencia de personalidades de la radio en las menciones de radio de los complementos alimenticios?

\subsection{Declaraciones de propiedades saludables en complementos alimenticios}

El marco regulatorio europeo comprende tres tipos de H-RCs (Parlamento y Consejo Europeo, 2000, 2002, 2006) y su aceptación en los siguientes términos: (1) 'declaraciones de enfermedades', que no están permitidas, promueven la noción de que ciertos tipos de alimentos previenen, tratan o curan una enfermedad humana; (2) las "declaraciones de función" están permitidas exclusivamente si se basan en pruebas científicas y están autorizadas por la Autoridad Europea de Seguridad Alimentaria (EFSA); (3) Podrían realizarse "declaraciones de reducción del riesgo de enfermedad" cuando lo autorice la EFSA a través del Proceso para la evaluación del apoyo científico a las declaraciones de propiedades sobre alimentos (PASSCLAIM) y se incorporen en una lista comunitaria.

En la televisión y los periódicos, las afirmaciones más frecuentes en los anuncios de suplementos dietéticos estaban relacionadas con la salud en general (40\%), eran específicas de la enfermedad $(38,8 \%)$, terapéuticas $(17,6 \%)$ o tenían fines cosméticos, sexuales o para bajar de peso $(14,7 \%)$ (Lee 
et al., 2015). Sin embargo, las declaraciones de propiedades saludables prohibidas para los complementos alimenticios estaban presentes en el 8,5\% de los sitios web checos (Baudischova et al., 2018), y el 84,5\% de los anuncios en los periódicos mencionaban hasta 12 declaraciones diferentes de enfermedades para cada producto (Chung et al., 2007).

Los trabajos de Soller et al. (2007) y Ethan et al. (2016) mostraron que las "afirmaciones de función" son los H-RCs más prevalentes en los anuncios de suplementos dietéticos en revistas, con una presencia del 58\% y 79\%, respectivamente. Recientemente, Avery et al. (2017) concluyeron que el $87 \%$ de los anuncios de suplementos dietéticos en revistas incluían "afirmaciones de función" que se refieren a las enfermedades más graves, como la degeneración de la función cerebral, enfermedades cardíacas o cáncer. Del mismo modo, Hassali et al. (2012) observaron que el 13,3\% de los anuncios en revistas incluían afirmaciones relacionadas con la mejora y potenciación de la salud sexual.

Por lo tanto, basado en investigaciones anteriores, el análisis de los H-RCs en un medio masivo que hasta ahora ha recibido poca consideración tiene como objetivo llenar parcialmente este vacío de investigación. Por tanto, parece relevante averiguar cómo se relaciona el uso de declaraciones de H$\mathrm{RCs}$ con la presencia de personalidades de radio, lo que lleva a la siguiente pregunta:

$\mathbf{P I}_{2}$ : ¿Con qué frecuencia las personalidades de la radio mencionan los diferentes tipos de $\mathrm{H}-\mathrm{RCs}$ en las menciones de radio de los complementos alimenticios?

Algunos estudios (Ethan et al., 2016) han revelado que la mayoría de los comerciales de suplementos dietéticos no mencionan el ingrediente principal (79\%), mientras que otros (Lee et al., 2015) han concluido que mencionan un ingrediente reconocible del producto anunciado $(91,1 \%)$, que contenía dos o más elementos. Hassali et al. (2012) han demostrado que, en las revistas femeninas, los ingredientes más prevalentes fueron las vitaminas $(17,8 \%)$, las proteínas $(10,2 \%)$ y los minerales $(9,6 \%)$. El trabajo de Baudischova et al. (2018) también mostró una aparición significativa de ingredientes no herbales, vitaminas y minerales, mientras que los ingredientes herbales se mencionan en el 70,1\% de los casos (Lee et al., 2015). Además, Philen et al. (1992) detectaron que los aminoácidos eran el ingrediente mencionado más recurrente $(21,7 \%)$ en las revistas de fisicoculturismo. En la medida en que todos los H-RCs deben ser veraces y la Directiva europea sobre complementos alimenticios solo permite minerales y vitaminas (Parlamento y Consejo Europeo, 2002), sería interesante saber cómo las personalidades de la radio utilizan esta información del producto, lo que lleva a la siguiente pregunta de investigación:

$\mathbf{P I}_{3}$ : ¿Con qué frecuencia las personalidades de la radio mencionan los ingredientes de los productos en las menciones de radio de los complementos alimenticios?

\section{Metodología}

Según lo declarado por la Asociación Americana de Mercadeo (AMA), una declaración publicitaria es una afirmación sobre los beneficios, las propiedades y/o el desempeño de un producto o servicio, cuyo objetivo es persuadir al cliente potencial. Así, la naturaleza empírica de este estudio parece requerir un análisis de los enunciados verbales, como los elementos más relevantes del mensaje y descripción del producto. En este caso, se han seleccionado menciones de radio para su análisis y codificación.

En línea con esta decisión, la metodología elegida para desarrollar este trabajo sigue un enfoque cuantitativo basado en el análisis de contenido que permite la descripción objetiva y analítica de todas las menciones radiofónicas emitidas a lo largo de 2017 en las emisoras de radio de 
noticias/entrevistas de España, con contenidos de programación basados en noticias y actualidad. La selección de las estaciones siguió dos criterios: cobertura nacional y transmisión en español. De acuerdo con el Estudio General de Medios (EGM) (AIMC, 2017), las estaciones de radio con mayor audiencia son: Cadena Ser, Cadena Cope y Onda Cero, con 9.000.000 de oyentes diarios.

Los datos analizados se obtuvieron de la base de datos de Arce Media (incorporada desde 2007 a la base de datos de Nielsen), empresa dedicada a la recopilación y análisis de publicidad en medios convencionales. Mediante un software específico, la empresa monitorea y registra toda la publicidad radial transmitida en diferentes formatos. Los autores han comprado el acceso a la base de datos y, por tanto, a las menciones de la categoría de producto requerida emitidas en el año de referencia en formato MP3. En este estudio, los complementos alimenticios pertenecen a un grupo de no medicamentos dentro de la categoría de salud e incluyen los siguientes tipos de productos: complementos alimenticios y vitamínicos, potenciadores de energía, suplementos para adelgazar, tónicos, suplementos de regeneración celular y otros productos nutricionales. Con base en este criterio de selección, el corpus de análisis incluye 437 menciones radiales, lo que brinda la oportunidad de trabajar con el universo completo. Todo el proceso de análisis y codificación fue realizado por dos codificadores que realizaron su trabajo de acuerdo a las siguientes variables y atributos:

1. Emisoras de radio de noticias/entrevistas: (1) Cadena Ser; (2) Cadena Cope; (3) Onda Cero.

2. Rol de la personalidad de la radio. Nivel de responsabilidad de la personalidad de la radio dentro del programa. La codificación se centra en la voz que describe el producto: (1) Presentador de radio: el director del programa es un periodista de renombre, responsable de la ejecución del programa. Como líder de opinión pública, tiene una gran influencia en su audiencia y asume los roles de editor, columnista y comentarista; (2) Colaborador: Otros periodistas, comentaristas o columnistas que colaboran como invitados en determinadas secciones fijas del programa; (3) Ambos: Locutor de radio y colaboradores que intervienen en la descripción del producto.

3. Tipos de categoría de declaraciones de propiedades saludables. Categoría no exclusiva, ya que se pueden incorporar una o más declaraciones en un anuncio. La conceptualización de las variables se basó en la normativa de la UE:

- Declaraciones de enfermedades (DE). Se refiere a la prevención, el tratamiento o la cura de una enfermedad. La declaración se refiere a los efectos del producto sobre una enfermedad, con una afirmación explícita (por ejemplo, la marca " $\mathrm{X}$ " mejora la artritis) o una afirmación implícita (por ejemplo, la marca "Y" alivia el dolor articular): (1) Ausencia; (2) Presencia.

- Declaraciones de funciones (DF). Referir o describir el papel de los nutrientes u otras sustancias en el desarrollo, crecimiento y funciones del cuerpo; en funciones psicológicas y conductuales; y en el control de peso o reducción del hambre. Dos ejemplos de afirmaciones autorizadas por la EFSA son: El calcio mantiene los huesos en buen estado y ayuda a la función muscular y la neurotransmisión adecuadas: (1) Ausencia; (2) Autorizada; (3) No autorizada.

- Declaraciones de reducción del riesgo de enfermedad (DRRE). Afirmar o sugerir que el consumo de un suplemento o de uno de sus componentes reduce significativamente un factor de riesgo en el desarrollo de una enfermedad. Un ejemplo de afirmación autorizada por la EFSA en base al mineral mencionado es: El calcio ayuda a reducir la pérdida de mineral óseo en mujeres posmenopáusicas. La baja densidad mineral ósea es un factor de riesgo de fracturas óseas osteoporóticas: (1) Ausencia; (2) Autorizada; (3) No autorizada.

4. Categoría de información de contenido del producto. Los ingredientes y sustancias del producto que se mencionan. Categoría no exclusiva, ya que se pueden incluir una o más 
declaraciones en un anuncio. La conceptualización de las variables se basó en la normativa de la UE:

- Vitaminas: (1) Ausencia; (2) Autorizada; (3) No autorizada.

- Minerales: (1) Ausencia; (2) Autorizada; (3) No autorizada.

- Otras sustancias: (1) Ausencia; (2) Autorizada; (3) No autorizada.

Para codificar si entre los tipos de H-RCs y las categorías de información del contenido del producto, una declaración de función, una declaración de reducción del riesgo de enfermedad, un ingrediente o una sustancia han sido autorizados por la EFSA, se validó con el sitio web del registro de la UE sobre declaraciones de propiedades nutricionales y saludables (Comisión Europea, 2016).

La confiabilidad inter-codificadora se midió mediante el método Kappa de Cohen, que plantea una variación entre 0,785 y 1, calculado con SPSS (versión 17). Además de las variables estructurales Estación de Radio y Franja Horaria $(\mathrm{k}=1)$, la variable Papel de Personalidad de Radio también obtuvo un valor de $\mathrm{k}=1$. En cuanto a las variables incluidas en la categoría Tipos de declaraciones de propiedades saludables, para las declaraciones de enfermedad $\mathrm{k}=0,785$, para las declaraciones de función $\mathrm{k}=1$ y para las declaraciones de reducción de riesgo de enfermedad k=0,955. Finalmente, $\mathrm{k}=1$ en el caso de todas las variables que integran la categoría de información de contenido de Producto, como Vitaminas, Minerales y Otros. Para solucionar las pocas divergencias detectadas, se realizó una tercera sesión de trabajo. Después de evaluar los casos, los dos codificadores decidieron la codificación final. Los resultados que se muestran a continuación se basan en un valor k=1 para todas las variables. Además, todos los datos cruzados de las variables codificadas se han sometido a pruebas de significación estadística relevantes mediante un análisis $\chi^{2}$ no paramétrico.

\section{Resultados}

Los primeros resultados indican varios aspectos significativos que contextualizan efectivamente el tema de estudio de nuestra investigación. En primer lugar, como se muestra en la tabla 1, cabe señalar que, de las tres emisoras de radio analizadas, la más importante en cuanto a índices de audiencia (Cadena Ser) evita el uso de personalidades radiofónicas para publicitar complementos dietéticos o alimenticios que conlleven implicaciones para la salud de los oyentes. Este tipo de emisiones se concentran principalmente en Cadena Cope $(76,9 \%)$ y en menor medida en Onda Cero $(23,1 \%)$. La primera, Cadena Cope, tiene un uso más intensivo de las personalidades radiales estudiadas. De hecho, acumula el 75,8\% de los locutores de radio y el 78,2\% de los colaboradores analizados en la muestra estudiada. Onda Cero, también utiliza estos recursos publicitarios, aunque en menor medida. Concentra el $24,2 \%$ y el $21,8 \%$ de la publicidad con locutores de radio y colaboradores, respectivamente.

Tabla 1. Personalidades de radio y estaciones de radio de noticias/entrevistas.

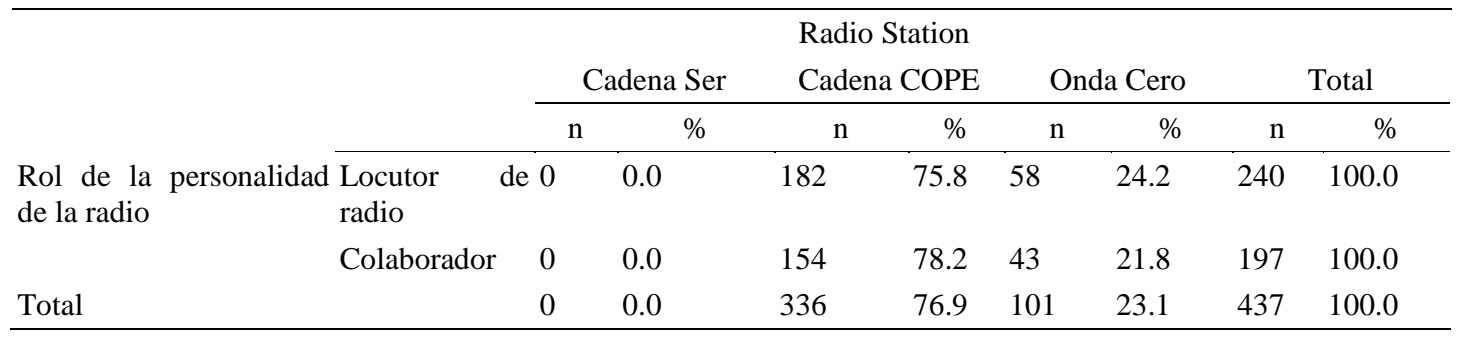

Fuente: Elaboración propia.

Nota: rol de la personalidad de la radio y estación de radio; $\chi^{2}: .333$; Significado: .564 
Respondiendo a la PI1, los resultados obtenidos del análisis de los 437 patrocinios muestran una presencia relativamente equilibrada de los dos roles de personalidades de la radio, predominando locutores de radio con 55\% de presencia (240) sobre el $45 \%$ de presencia (197) de colaboradores. El atributo Ambos no tiene presencia ya que, en la práctica real, la descripción del producto suele ser expresada en su totalidad por una de las dos personalidades de la radio.

Analizar la frecuencia con la que las personalidades de radio mencionan los diferentes tipos de $\mathrm{H}$ RCs es uno de los principales objetivos de este trabajo. La Tabla 2 responde parcialmente a la PI 2 y muestra la relación entre la variable personalidad de la radio y declaraciones de enfermedad (DE), que, como se mencionó anteriormente, están prohibidas en la publicidad de complementos alimenticios. Sin embargo, el 20,4\% (89) del total de menciones radiales utilizan afirmaciones que se refieren explícita o implícitamente a la prevención o tratamiento de enfermedades a través del consumo de este tipo de productos, y de este porcentaje, el 77,5\% están avalados por colaboradores y el 22,5\% por locutores de radio. Los siguientes extractos proporcionan ejemplos de declaraciones de enfermedades detectadas en la muestra en estudio: "[La marca X disminuye el dolor en las articulaciones y le ayuda a recuperar su calidad de vida]"; "[Durante los primeros síntomas de pérdida de memoria]".

Tabla 2. Personalidades de la radio y declaraciones de enfermedades.

\begin{tabular}{llrrrrrr}
\hline & \multicolumn{5}{c}{ Declaraciones de enfermedades } \\
& & Ausencia & Presencia & \multicolumn{2}{c}{ Total } \\
\cline { 2 - 7 } & & $\mathrm{n}$ & $\%$ & $\mathrm{n}$ & $\%$ & $\mathrm{n}$ & $\%$ \\
\hline Rol de la personalidad de la radio & Locutor de radio & 220 & 91.7 & 20 & 8.3 & 240 & 100.0 \\
& Colaborador & 128 & 65.0 & 69 & 35.0 & 197 & 100.0 \\
Total & & 348 & 79.6 & 89 & 20.4 & 437 & 100.0 \\
\hline
\end{tabular}

Fuente: Elaboración propia.

Nota: el rol de la personalidad de la radio y las declaraciones de enfermedades; $\chi^{2}: 47.528$; Significado: .001

La Tabla 3 proporciona la respuesta final a PI2 y relaciona los otros dos tipos de H-RCs y la variable rol de la personalidad de la radio. Las declaraciones de función son el tipo de declaraciones más frecuentes en las menciones radiales de complementos alimenticios, aunque la mayoría no han sido autorizadas. Específicamente, las declaraciones de función ilícitas se mencionan en el 66.7\% (240) de los patrocinios expresadas por los presentadores de radio y en el 97\% expresadas por los colaboradores. Los siguientes extractos proporcionan ejemplos de cómo varios colaboradores respaldan el mismo producto en diferentes anuncios utilizando declaraciones de Función: "[La marca $\mathrm{X}$ ayuda a disminuir el cansancio y la fatiga y le ayuda a recuperar su metabolismo energético y vitalidad]"; "[La marca X proporciona los nutrientes, vitaminas y minerales necesarios para tener energía y vitalidad durante todo el día]"; "[Ayudan a cuidar y proteger sus articulaciones]".

Por otro lado, el uso de Declaraciones de Reducción de Riesgo de Enfermedad presenta un peso relativo de solo el $8,7 \%$ en el corpus analizado. Ninguno de estos reclamos ha sido autorizado, y son expresados principalmente por colaboradores $(11,2 \%)$, aunque la presencia de locutores de radio también es relevante en el 6,7\% de los casos. El siguiente ejemplo presenta a un presentador de radio que se dirige a la audiencia en primera persona — estilo testimonial — para describir las propiedades saludables del producto, aunque sin mencionar ningún tipo de sustancia: "[La marca $X$ reduce y normaliza los niveles de colesterol malo]". 
Tabla 3. Personalidades de la radio, declaraciones de funciones $(D F)$ y declaraciones de reducción de riesgo de enfermedad (DRRE).

\begin{tabular}{|c|c|c|c|c|c|c|c|c|c|c|c|c|c|c|c|c|}
\hline & \multicolumn{8}{|c|}{ Locutor de radio } & \multicolumn{8}{|c|}{ Colaborador } \\
\hline & \multicolumn{2}{|c|}{ Ausencia } & \multicolumn{4}{|c|}{ Presencia } & \multirow{2}{*}{\multicolumn{2}{|c|}{ Total }} & \multirow{2}{*}{\multicolumn{2}{|c|}{ Ausencia }} & \multicolumn{4}{|c|}{ Presencia } & \multirow{2}{*}{\multicolumn{2}{|c|}{ Total }} \\
\hline & & & \multicolumn{2}{|c|}{ Autorizada } & \multicolumn{2}{|c|}{ No-Autorizada } & & & & & \multicolumn{2}{|c|}{ Autorizada } & \multicolumn{2}{|c|}{ No-Autorizada } & & \\
\hline & $\mathrm{n}$ & $\%$ & $\mathrm{n}$ & $\%$ & $\mathrm{n}$ & $\%$ & $\mathrm{n}$ & $\%$ & $\mathrm{n}$ & $\%$ & $\mathrm{n}$ & $\%$ & $\mathrm{n}$ & $\%$ & $\mathrm{n}$ & $\%$ \\
\hline DFs & 2 & 0.8 & 78 & 32.5 & 160 & 66.7 & 240 & 100.0 & 0 & 0.0 & 6 & 3.0 & 191 & 97.0 & 197 & 100 \\
\hline DRREs & 224 & 93.3 & 0 & 0.0 & 16 & 6.7 & 240 & 100.0 & 175 & 88.8 & 0 & 0.0 & 22 & 11.2 & 197 & 100 \\
\hline
\end{tabular}

Fuente: Elaboración propia.

Nota: Rol de la personalidad de la radio y declaraciones de función (DF); $\chi^{2}: 62.829$; Significado: .001 ; Rol de la personalidad de la radio y declaraciones de reducción de riesgo de enfermedad (DRRE); $\chi^{2}: 2.761$; Significado: .097

Todas las declaraciones de propiedades saludables relativas a los complementos alimenticios deben estar respaldadas por evidencia científica basada en una vitamina, mineral u otro tipo de sustancia autorizada. Así, en respuesta a la PI3, la Tabla 4 muestra la información proporcionada por personalidades de radio con respecto a los productos respaldados. El 78,9\% (345) de las menciones radiales de complementos alimenticios no mencionan - como se requiere - un mineral que sustente los supuestos beneficios del consumo del producto, y este porcentaje alcanza el 67,7\% (296) en el caso de vitaminas y el 56,2\% (246) en el caso de otras sustancias. Sin embargo, los minerales son el ingrediente mencionado con más frecuencia en las declaraciones autorizadas de función y reducción del riesgo de enfermedad expresadas por los locutores de radios $(23,4 \%)$ y colaboradores $(3 \%)$. El siguiente es un ejemplo de dos declaraciones de función autorizadas promovidas por un locutor de radio, que se refieren a dos minerales diferentes: '[Desarrollado en colaboración con el CSIC, contiene zinc, que contribuye a la síntesis efectiva de ADN, y selenio, que contribuye a la protección celular en casos de estrés oxidativo]'.

Por otro lado, en los H-RCs elaborados por colaboradores $(53,8 \%)$ y locutores de radio $(35,4 \%)$ predominan otras sustancias no autorizadas, seguidas de las vitaminas, que también son frecuentemente mencionadas por los colaboradores $(43,7 \%) \mathrm{y}$, en menor medida, por locutores de radio (17,9\%). El siguiente ejemplo describe un beneficio que la vitamina no puede proporcionar: "[La memoria es donde notas por primera vez tu edad. Te vuelves olvidadizo y tu mente parece no funcionar. Contiene fósforo y vitaminas del grupo B, que ayudan a fortalecer nuestra memoria]'.

Tabla 4. Personalidades de la radio e información sobre el contenido del producto.

\begin{tabular}{|c|c|c|c|c|c|c|c|c|c|c|c|c|c|c|c|c|}
\hline & \multicolumn{8}{|c|}{ Locutor de radio } & \multicolumn{8}{|c|}{ Colaborador } \\
\hline & \multirow{2}{*}{\multicolumn{2}{|c|}{ Ausencia }} & \multicolumn{4}{|c|}{ Presencia } & \multirow{2}{*}{\multicolumn{2}{|c|}{ Total }} & \multirow{2}{*}{\multicolumn{2}{|c|}{ Ausencia }} & \multicolumn{4}{|c|}{ Presencia } & \multirow{2}{*}{\multicolumn{2}{|c|}{ Total }} \\
\hline & & & \multicolumn{2}{|c|}{ Autorizada } & \multicolumn{2}{|c|}{$\begin{array}{c}\text { No- } \\
\text { Autorizada }\end{array}$} & & & & & & rizada & Aut & izada & & \\
\hline & $\mathrm{n}$ & $\%$ & $\mathrm{n}$ & $\%$ & $\mathrm{n}$ & $\%$ & $\mathrm{n}$ & $\%$ & $\mathrm{n}$ & $\%$ & $\mathrm{n}$ & $\%$ & $\mathrm{n}$ & $\%$ & $\mathrm{n}$ & $\%$ \\
\hline Vitaminas & 185 & 77.1 & 12 & 5.0 & 43 & 17.9 & 240 & 100 & 111 & 56.3 & 0 & 0.0 & 86 & 43.7 & 197 & 100 \\
\hline Minerales & 182 & 75.8 & 56 & 23.4 & 2 & 0.8 & 240 & 100 & 163 & 82.7 & 6 & 3.0 & 28 & 14.3 & 197 & 100 \\
\hline Otras & 155 & 64.6 & 0 & 0.0 & 85 & 35.4 & 240 & 100 & 91 & 46.2 & 0 & 0.0 & 106 & 53.8 & 197 & 100 \\
\hline
\end{tabular}

Fuente: Elaboración propia.

Nota: rol de la personalidad de la radio y vitamina; $\chi^{2}$ : 40.999; Significado: .001; Rol de personalidad de radio y Mineral; $\chi^{2}$ : 60.255; Significado: .001; Rol de la personalidad de la radio y otras sustancias; $\chi^{2}: 14.872$; Significado: .001 
Finalmente, y tomando en consideración los datos proporcionados en la Tabla 4, cabe señalar que ningún locutor de radio o colaborador ha mencionado algún H-RC autorizado respaldado por otras sustancias distintas de vitaminas o minerales.

\section{Discusión}

Esta investigación aporta nuevos datos sobre la publicidad de complementos alimenticios en el contexto europeo, donde los estudios de esta naturaleza son escasos. La principal contribución de este análisis es establecer la relación entre las personalidades de la radio como fuente creíble y la información del producto en los complementos alimenticios, que se consideran un producto de alta implicación para los consumidores debido a los problemas de salud que implican. Otra contribución de este estudio tiene que ver con la elección del medio analizado. La programación radial se basa en noticias de distinta índole, y su audiencia está predispuesta y acostumbrada a escuchar contenidos informativos. De hecho, la radio está determinada por la mayoría de españoles y europeos como la fuente de información más fiable (Comisión Europea, 2021).

Los locutores de radio suelen ser periodistas reconocidos responsables de la conducción del programa y, como líderes de opinión, tienen una gran influencia en sus audiencias. Los anunciantes utilizan locutores de radio — considerados celebridades (McCracken, 1989) — porque aumentan la efectividad publicitaria al proyectar en el producto los valores y la credibilidad de su profesión. Este trabajo confirma la prevalencia de este tipo de personalidades radiofónicas cuya información y credibilidad profesional percibida también se proyecta sobre el resto de mensajes (McCroskey \& Young, 1981), incluidos los mensajes publicitarios. Cuando un locutor de radio respalda un producto para una audiencia fiel que escucha diariamente su programa, los oyentes tenderán a aceptar los argumentos como válidos, sin cuestionar la confiabilidad del anuncio y dando por sentados los supuestos beneficios y propiedades del producto respaldado (Wong et al., 2020). Cuando el locutor de radio también reconoce su propio consumo del producto, esta experiencia se suma a la confiabilidad percibida — vinculada a la honestidad de la fuente (Kim y Yang, 2019; McGinnies y Ward, 1980; Mospan y Alexander, 2018) — aumentando la capacidad de persuasión del patrocinio (Wong et al., 2020). La confianza generada en los oyentes por locutores de radio y periodistas de renombre como líderes de opinión puede ser la principal razón por la que los anunciantes eligen este tipo de roles para promover su producto.

Con respecto al análisis de H-RCs, conviene recordar que los diferentes tipos de declaraciones no son mutuamente excluyentes, por lo que una mención puede incluir varios tipos. En este sentido, uno de cada cinco avales contiene declaraciones de enfermedad, práctica que también ha sido analizada en estudios previos (Baudischova et al., 2018; Chung et al., 2007; Lee et al., 2015). En conjunto, el análisis realizado muestra que si bien en este tipo de reclamos ilícitos — cuyo uso está prohibidolas personalidades de la radio no mencionan directamente enfermedades, sí lo hacen de manera indirecta. Por lo tanto, se refieren explícitamente al envejecimiento como la causa del dolor articular o la pérdida de memoria en lugar de nombrar artrosis, Alzheimer o demencia senil. Adicionalmente, este tipo de reclamo no solo propone la curación de una enfermedad o la desaparición inmediata del dolor, también recomienda el consumo de un producto para su prevención, como en el siguiente ejemplo: '[A medida que envejeces, notas cómo pierde memoria y agilidad mental... La marca X contiene una alta concentración de fosfolípidos, vitaminas y minerales que conservan tu memoria; es el complemento indicado para evitar la pérdida de memoria]'. Las apelaciones de miedo y la mención de términos médicos o clínicos — como "síntomas" - o factores de riesgo que pueden empeorar nuestra calidad de vida — como el envejecimiento - también son frecuentes en las menciones radiofónicas de los complementos alimenticios. 
Este estudio aporta datos empíricos novedosos sobre la fuente que avala este tipo de productos, demostrando que los colaboradores prevalecen en las declaraciones de enfermedades sobre los locutores de radio. Ambos, en ocasiones, asumen el papel de prescriptores como si fueran médicos que asesoran sobre la dosis y uso del producto: "[Dos cápsulas al día de la marca X alivia el dolor]; [dos cápsulas al día son suficientes]; [una cápsula al día reduce y normaliza los niveles de colesterol malo]". En este sentido, cuando las personalidades de la radio respaldan productos emitiendo opiniones fuera de su área de especialización, la información proporcionada podría estar sesgada y engañar a los consumidores.

De acuerdo con estudios previos (Avery et al., 2017; Ethan et al., 2016; Soller et al., 2007), las declaraciones de función tienen una alta presencia en la publicidad de suplementos dietéticos. El presente trabajo confirma firmemente este hallazgo. Las afirmaciones de función expresadas predominantemente por locutores de radio aparecen en casi todos los casos analizados. De estos, más de la mitad no han sido autorizados por la EFSA. Es una práctica habitual en las menciones de radio de los complementos alimenticios describir los beneficios para la salud como atributos del producto: "[La marca X es el único producto que normaliza el colesterol]; [La marca Y está específicamente formulado para cuidar y nutrir sus huesos y articulaciones, y prevenir el dolor, porque no se puede evitar envejecer]; [La marca $\mathrm{Z}$ cuida tu memoria]". Sin embargo, las declaraciones solo deben hacer referencia al papel de los nutrientes u otras sustancias en términos de crecimiento, desarrollo y funciones del cuerpo para el mantenimiento de una buena salud. Además, otras afirmaciones hechas por personalidades de la radio aseguran a los oyentes los supuestos beneficios del producto: "[La marca X es la solución]; [La marca X es un revitalizador de efectividad garantizada]'- apelar a una '[fórmula nueva y más poderosa]', '[fórmula exclusiva]', '[fórmula natural]', '[fórmula efectiva probada por laboratorios profesionales]', o denotar que el producto se vende en farmacias. Estos recursos publicitarios pueden condicionar a los consumidores a tomar una decisión de compra influenciada por la omisión de información indispensable sobre el producto. De esta forma, la confianza de los oyentes en las personalidades de la radio se convierte en un factor decisivo de gran persuasión (Martín-Santana et al., 2017).

En cuanto a la información que brindan las personalidades de la radio en relación al contenido de los complementos alimenticios, y nuevamente en concordancia con estudios previos (Ethan et al., 2016), este trabajo muestra que la mayoría de las menciones radiales analizadas no nombran ningún tipo de sustancia obligatoria exigida por la legislación vigente. Otras sustancias son el tipo más mencionado, aunque las declaraciones en las que se incluyen no han sido autorizadas. En contraste con investigaciones anteriores que encontraron que las sustancias mencionadas con mayor frecuencia eran las vitaminas (Baudischova et al., 2018; Hassali et al., 2012), en este estudio las vitaminas ocupan el segundo lugar, aunque, nuevamente, en la mayoría de los casos su uso es ilícito. Finalmente, y a pesar de ser las sustancias con menor presencia, los minerales acumulan el mayor número de declaraciones autorizadas. En general, las declaraciones autorizadas que se refieren a las diferentes sustancias analizadas son expresadas con mayor frecuencia por locutores de radio, mientras que los colaboradores utilizan en mayor medida declaraciones no autorizadas en menciones de radio donde describen el producto. El uso de argumentos vagos y generales como '[La marca X aporta vitaminas, minerales y nutrientes]' es frecuente en las menciones radiales de complementos alimenticios y, aunque su presencia ha quedado registrada en el proceso de codificación, no puede considerarse autorizada ya que los argumentos no señalan la sustancia ni declaran el beneficio para la salud que brindan.

La publicidad, como forma de comunicación masiva, llega e informa a los consumidores de la existencia y disponibilidad de productos en el mercado con rapidez y eficiencia. Sin embargo, las 
malas prácticas en la promoción de complementos alimenticios —afirmaciones falsas, ilícitas y engañosas, o la omisión de características esenciales y/o beneficios de los productos - pueden llevar a los consumidores a decisiones de compra equivocadas (Kowalska et al., 2019). Es importante tener en cuenta que el reglamento actual (Parlamento y Consejo Europeo, 2006) establece como principio general que las declaraciones de propiedades saludables no deben ser engañosas, confusas o inciertas. En este sentido, los anunciantes explotan la confiabilidad de los líderes de opinión y utilizan personalidades de la radio para promocionar los complementos alimenticios, a veces de manera testimonial mediante la cual comparten su experiencia de consumo con la audiencia (Kim \& Yang, 2019; Muela-Molina et al., 2020). Algunos locutores de radio incluso recomiendan el producto como si fueran médicos, engañando a los consumidores para que piensen en los complementos alimenticios como una alternativa a los medicamentos o la medicina tradicional, lo que a su vez promueve la práctica del autocuidado en lugar del asesoramiento profesional que a menudo se requiere (Mospan \& Alexander, 2018). En el contexto de un mercado en rápida expansión, la demanda de este tipo de productos está aumentando a nivel mundial (Binns et al., 2018), mientras que la legislación vigente —que es relativamente reciente- y su aplicación siguen siendo inadecuadas.

\subsection{Implicaciones de gestión}

La publicidad de complementos alimenticios está sujeta a controversia ya que proporciona información sobre productos que pueden suponer un riesgo para la salud o implicar un fraude económico (Baudischova et al., 2018). La preocupante presencia de H-RCs ilícitos ha sido reconocida como un riesgo público en la medida en que induce falsas percepciones y comportamientos inapropiados (Royne et al., 2016). Numerosos expertos e investigadores (Binns et al., 2018; Kowalska et al., 2019; Starr, 2016) ya han expresado su preocupación, así como exigido la necesidad de una legislación restrictiva en la publicidad de complementos alimenticios y un estricto seguimiento de los fabricantes, teniendo en cuenta la laxitud administrativa, problemas de salud y otros problemas observados desde la aplicación del texto legal vigente (Binns et al., 2018; Makowska \& Jasiński, 2019; Starr, 2016).

Estudios recientes despiertan preocupación por la gran presencia de H-RCs engañosos (Baudischova et al., 2018; Lee et al., 2015) e incluso de declaraciones audaces que carecen de cualquier tipo de soporte científico (Royne et al., 2016; Silchenko \& Askegaard, 2020) o que no son creíbles (Plasek \& Temesi, 2019; Soller et al., 2007). Aunque el objetivo principal de la regulación de la UE es evitar la existencia de declaraciones engañosas, nuestra investigación ha encontrado frecuentes declaraciones vagas que pueden considerarse claramente engañosas por omisión (Kowalska et al., 2019). Por lo tanto, dado el alto nivel de consumo y publicidad de este tipo de productos, sería beneficioso implementar mecanismos relevantes para proteger a los consumidores, tales como: supervisión gubernamental eficiente de la actividad publicitaria; un aumento de las sanciones impuestas por los sistemas de autorregulación; y, en los medios, la implementación de un sistema de autorización previa que asegure que los mensajes estén en sintonía con los requisitos legales antes de su difusión. Esto filtraría las comunicaciones engañosas antes de que lleguen a los consumidores, y así evitaría la compra de productos que no cumplen con los beneficios anunciados.

Por otro lado, los locutores y colaboradores de radio pueden engañar a los consumidores cuando emiten opiniones sobre un tema fuera de su área de especialización, de modo que el uso de endosos obliga al conocimiento y comprensión de los parámetros legales asociados. Aunque las personalidades de la radio generalmente carecen de los conocimientos calificados para respaldar los complementos alimenticios, los anunciantes los usan con frecuencia. El caso de los periodistas y locutores de radio como prescriptores es especial por su influencia social y reconocimiento como 
líderes de opinión pública. Adicionalmente, el código ético del sector no permite su participación en ningún mensaje o actividad publicitaria (Muela-Molina et al., 2020). En este sentido, los anunciantes son los responsables en última instancia de su publicidad y contenido, por lo que cuando se infringe la ley o los códigos, los prescriptores - la fuente del mensaje - suelen estar exentos de cualquier responsabilidad. Esta impunidad debe ser analizada en términos de política pública, ya que los prescriptores también deben ser responsables de lo que dicen a los consumidores. Asimismo, debido a que la información de salud puede estar relacionada con el riesgo representativo y los consumidores pueden otorgar mayor confianza a un respaldo de una fuente confiable (Rollins et al, 2020; Royne et al., 2016), las personalidades de la radio y los líderes de opinión pública deben mostrar una mayor responsabilidad hacia la sociedad y un mayor respeto de los códigos y la ley. Y finalmente, los medios de comunicación en general - en este caso el medio radial- deberían implementar un sistema de pre-autorización dentro del marco de autorregulación para evitar la difusión de prescripciones ilícitas.

\subsection{Limitaciones y futura investigación}

Este trabajo se ha centrado en el medio radial y las estaciones de servicio completo. El alcance de la investigación futura podría ampliarse para incluir otros tipos de estaciones de radio y medios, como la televisión. En este sentido, sería pertinente comparar la presencia de personalidades de la radio en las menciones radiales de complementos alimenticios en diferentes países europeos, para una mejor comprensión del impacto de los determinantes culturales y sociológicos, ya que todos compartimos los mismos principios legales.

Otra limitación de este trabajo es que solo analiza la presencia de H-RCs y sustancias permitidas por la ley en las menciones radiofónicas de los complementos alimenticios. Es decir, el siguiente paso requeriría un análisis más especializado del objeto de estudio desde la perspectiva de la autorregulación. También sería relevante realizar un análisis desde la perspectiva de los consumidores para determinar su conocimiento sobre los diferentes tipos de H-RCs y si son capaces de distinguirlos.

Los resultados de este trabajo allanan el camino para el desarrollo de futuras investigaciones experimentales que puedan dar continuidad o resolver interrogantes e hipótesis emergentes. Por ejemplo, sería interesante saber cómo la confiabilidad de la fuente afecta el procesamiento de la información y en qué medida el poder persuasivo del mensaje depende del género y el rol de la personalidad de la radio - ya sea hombre o mujer, embajador o testimonial.

También cabe señalar que los resultados de este trabajo muestran que una de las tres estaciones de radio analizadas no emitió menciones de complementos alimenticios. Esto requiere un análisis más profundo que explique las razones detrás de esto. Sería interesante entrevistar a los propietarios y locutores de radio para conocer mejor el proceso de gestión publicitaria, con énfasis en la aceptación, producción, revisión y difusión de menciones radiofónicas de complementos alimenticios. La implementación de sistemas de autorregulación y rendición de cuentas, la observación de la línea editorial o la existencia de una guía de estilo también abren líneas de investigación futura.

\section{Conclusiones}

El elevado uso de declaraciones de propiedades saludables no permitidas por la ley para la promoción de complementos alimenticios prescriptas por personalidades de la radio es motivo de preocupación. El hecho de que una de cada cinco menciones incluya declaraciones que aseguren la cura, alivio o prevención de una enfermedad explícitamente prohibida por la ley evidencia el 
comportamiento irresponsable de locutores y colaboradores de radio, quienes, en cualquier caso, no deben colaborar en campañas publicitarias, ya que no está permitido por el código ético de la profesión. Para agravar la situación, hay que destacar la presencia alarmante de H-RCs no autorizados que, en el caso de las declaraciones de función, aparecen en la mayoría de las menciones, mientras que ninguna de las declaraciones de reducción de riesgo de enfermedad analizadas ha sido autorizada por el marco regulatorio europeo y, por extensión, el marco regulatorio español. Además, con respecto a la información relacionada con los ingredientes de los productos que debe sustentar los beneficios para la salud prescriptos por las personalidades de la radio, en la mayoría de los casos se omite o es tan vaga y ambigua que induce a error. Esto también está incluido en el reglamento, que estipula que los H-RCs deben estar respaldados por sustancias y propiedades para la salud aprobadas por la EFSA.

Si bien, cuando se infringe la ley, los anunciantes son los únicos responsables de la prescripción ilícita, la responsabilidad social hacia los destinatarios del mensaje debe extenderse a los propietarios de los medios de comunicación y las personalidades de la radio, debido a la influencia social y al potencial poder de persuasión de ciertos prescriptores, como los presentadores de radio y/o periodistas como líderes de opinión. Además, la preocupante presencia de mensajes engañosos afirmaciones falsas y vagas, y omisión de información del producto - sugiere que las administraciones públicas y las autoridades autorreguladoras deben aplicar mecanismos de seguimiento y control más estrictos, así como sanciones más severas, a la publicidad de complementos alimenticios con el fin de disuadir a los anunciantes de nuevas infracciones de la ley y proteger a los consumidores. En el caso de la UE, parece fundamental abordar la legislación reguladora actual sobre publicidad de complementos alimenticios con un plan de acción y un marco común de sanciones, de modo que los responsables de las políticas gubernamentales de los estados miembros puedan proteger la salud de los consumidores de los efectos potencialmente peligrosos de su consumo, a menudo promovido a través de malas prácticas publicitarias. Los responsables de la formulación de políticas deberían responsabilizar a los medios y las personalidades de la radio como prescriptores, e incluso prohibir el uso de estos últimos en los anuncios de complementos alimenticios para proteger a los consumidores contra la publicidad ilícita relacionada con la salud.

\section{Bibliografía}

Asociación Española de Anunciantes (AEA). (2021). Datos clave 2020-2021 [Key Facts 2020-2021]. https://www.anunciantes.com/herramientas-aea/

Asociación para la Investigación de Medios de Comunicación (AIMC). (2017). Resumen General del EGM (Estudio General de Medios). [EGM (Media General Study) General Report]. https://www.aimc.es/a1mc-c0nt3nt/uploads/2017/05/resumegm317.pdf

Asociación para la Investigación de Medios de Comunicación (AIMC). (2021). 2021-1ª Ola. [2021$1^{\text {st }}$ Wave] https://reporting.aimc.es/index.html\#/main/cockpit

Avery, R. J., Eisenberg, M. D., \& Cantor, J. H. (2017). An examination of structure-function claims in dietary supplement advertising in the US: 2003-2009. Preventive Medicine, 97, 86-92. https://doi.org/10.1016/j.ypmed.2017.01.008

Baudischova, L., Straznicka, J., Pokladnikova, J., \& Jahodar, L. (2018). The quality of information on the internet relating to top-selling dietary supplements in the Czech Republic. International Journal of Clinical Pharmacy, 40(1), 183-189. https://doi.org/10.1007/s11096-017-0564-x 
RLCS, Revista Latina de Comunicación Social, 79, 263-282

[Investigación] DOI: 10.4185/RLCS-2021-1511| ISSN 1138-5820| Año 2021

Binns, C. W., Lee, M. K., \& Lee, A. H. (2018). Problems and prospects: public health regulation of dietary supplements. Annual Review of Public Health, 39, 403-420. https://doi.org/10.1146/annurev-publhealth-040617-013638

Chung, E. P., Hwang, H. J., \& Kim, M. K. (2007). Evaluation of non-English dietary supplement advertisements in an ethnic minority community in America. Public Health Nutrition, 10(8), 834837. https://doi.org/10.1017/s136898000733406x

Cornia, A., Sehl, A., \& Nielsen, R. K. (2018). 'We no longer live in a time of separation': A comparative analysis of how editorial and commercial integration became a norm. Journalism, 21(2), 172-190. https://doi.org/10.1177/1464884918779919

Dens, N., De Pelsmacker, P., \& Verhellen, Y. (2018). Better together? Harnessing the power of brand placement through program sponsorship messages. Journal of Business Research, 83, 151159. https://doi.org/10.1016/j.jbusres.2017.10.036

Espinosa Mirabet, S., \& Vico Blanco, M. (2016). La involución de los formatos comerciales radiofónicos: España 10 años después [Involution of the structure of radio advertisements: Spain ten years later]. ZER-Revista de Estudios de Comunicación, 21(41), 227-243. https://doi.org/10.1387/zer.17293

Ethan, D., Basch, C. H., Berdnik, A., \& Sommervil, M. (2016). Dietary supplements advertised in muscle enthusiast magazines: a content analysis of marketing strategies. International Journal of Men's Health, 15(2), 194-202. https://doi.org/10.3149/jmh.1502.194

European Commission. (2016, October 20). EU Register of Nutrition and Health Claims made on foods. http://ec.europa.eu/food/safety/labelling_nutrition/claims/register/public/?event=search

European Commission. (2021). Standard Eurobarometer 94. Winter 2020-2021. Public Opinion in the European Union. https://europa.eu/eurobarometer/surveys/detail/2355

European Parliament and Council. (2000). Directive 2000/13/EC of the European Parliament and of the Council of 20 March 2000 on the approximation of the laws of the Member States relating to the labelling, presentation and advertising of foodstuffs. https://eur-lex.europa.eu/legalcontent/EN/TXT/PDF/?uri=CELEX:32000L0013\&from=EN

European Parliament and Council. (2002). Directive 2002/46/EC of the European Parliament and of the Council of 10 June 2002 on the approximation of the laws of the Member States relating to food supplements (Text with EEA relevance). http://data.europa.eu/eli/dir/2002/46/2017-07-26

European Parliament and Council. (2006). Regulation (EC) No 1924/2006 of the European Parliament and of the Council of 20 December 2006 on nutrition and health claims made on foods. http://data.europa.eu/eli/reg/2006/1924/2014-12-13

FAPE (Federación de Asociaciones de Periodistas de España). (2017). Código Deontológico [Deontological Code]. Available at: http://fape.es/home/codigo-deontologico/

Ha, L. (2018). Advertising in Media Management and Economics. In A. Albarran, B. Mierzejewska, \& J. Jung, (Eds.). Handbook of media management and economics (pp. 144-158). Routledge. 
Hassali, M. A., Saleem, F., Aljadhey, H., \& Khan, T. M. (2012). Evaluating the content of advertisements for dietary supplements in Malaysian women's magazines. Drug Information Journal, 46, 723-728. https://doi.org/10.1177/0092861512457777

Kim, J. \& Yang, H. (2019). How Does a Radio Host's Testimonial Influence Media Experiences? The Indirect Effect of Social Presence. Journal of Radio \& Audio Media, 26(2), 336-350. https://doi.org/10.1080/19376529.2018.1509217

Kowalska, A., Bieniek, M., \& Manning, L. (2019). Food supplements' non-conformity in Europe Poland: A case study. Trends in Food Science \& Technology, 93, 262-270. https://doi.org/10.1016/j.tifs.2019.09.022

Lee, A., Vásquez, L. J., Wong, W. C., \& Shin, J. (2015). Evaluation of dietary supplement advertisements in popular Spanish, Chinese, and Korean media outlets: a cross sectional study. BMC Nutrition, 1(1), 43. https://doi.org/10.1186/s40795-015-0038-2

Makowska, M., \& Jasiński, Ł. (2019). A discussion of the unresolved 2016/17 plans for regulating the Polish dietary supplements market. Health Policy, 123(6), 544-549. https://doi.org/10.1016/j.healthpol.2019.04.001

Martín-Santana, J. D., Reinares-Lara, E., \& Reinares-Lara, P. (2017). How does the radio spokesperson's voice influence credibility? Communications, 42(2), 151-172. https://doi.org/10.1515/commun-2017-0015

McCracken, G. (1989). Who is the celebrity endorser? Cultural foundations of the endorsement process. Journal of Consumer Research, 16(3), 310-321. https://doi.org/10.1086/209217

McCroskey, J. C., \& Young, T. J. (1981). Ethos and Credibility: The Construct and its Measurement after three Decades. Central States Speech Journal, 32(1), 24-34. https://doi.org/10.1080/10510978109368075

McGinnies, E., \& Ward, C. D. (1980). Better liked than right trustworthiness and expertise as factors in credibility. Personalilty and Social Psychology Bulletin, 6(3), 467-472.

https://doi.org/10.1177/014616728063023

Moorman, M., Willemsen, L. M., Neijens, P. C., \& Smit, E. G. (2012). Program-involvement effects on commercial attention and recall of successive and embedded advertising. Journal of Advertising, 41(2), 25-38. https://doi.org/10.2753/JOA0091-3367410202

Morimoto, M. (2020). OTC drug advertising in Japan: the role of need for cognition and celebrity endorser credibility. Health Marketing Quarterly, 37(2), 108-123. https://doi.org/10.1080/07359683.2020.1742479

Mospan, C. M., \& Alexander K. M. (2018). Utilizing celebrity endorsements to teach over-thecounter medication and dietary supplement regulations. Currents in Pharmacy Teaching and Learning, 10(11), 1507-1511. https://doi.org/10.1016/j.cptl.2018.08.001

Muela-Molina, C., Martín-Santana, J. D., \& Reinares-Lara, E. (2020). Journalists as radio advertising endorsers in news or talk radio stations. Journalism, 21(12), 1913-1931. https://doi.org/10.1177/1464884917753785 
Muela-Molina, C., Perelló-Oliver, S., \& García-Arranz, A. (2021). Health-related claims in food supplements endorsements: a content analysis from the perspective of EU regulation. Public health, 190, 168-172. https://doi.org/10.1016/j.puhe.2020.10.020

Perelló-Oliver, S., \& Muela-Molina, C. (2017). Radio Mentions: An Analysis of Radio Personalities and Ethical Behaviour (Spain). International Journal of Communication, 11, 3566-3586.

Perelló-Oliver, S., Muela-Molina, C., \& Campos-Zabala, M. V. (2018). Brand personification in radio advertising. Analysis of the presence and use of the figure of the spokesperson. Revista Latina de Comunicación Social, 73, 1163-1178. http://dx.doi.org/10.4185/RLCS-2018-1301

Philen, R. M., Ortiz, D. I., Auerbach, S. B., \& Falk. H. (1992). Survey of advertising for nutritional supplements in health and bodybuilding magazines. Journal of the American Medical Association (JAMA), 268(8), 1008-1011. https://doi.org/10.1001/jama.1992.03490080082029

Plasek, B., \& Temesi, Á. (2019). The credibility of the effects of functional food products and consumers' willingness to purchase/willingness to pay- review. Appetite, 143, 104398. https://doi.org/10.1016/j.appet.2019.104398

Rollins, B., Huh, J., Bhutada, N., \& Perri, M. (2020). Effects of endorser type and testimonials in direct-to-consumer prescription drug advertising (DTCA). International Journal of Pharmaceutical and Healthcare Marketing, 15(1), 1-17. https://doi.org/10.1108/IJPHM-06-2019$\underline{0042}$

Royne, M. B., Myers, S. D., Deitz, G., \& Fox, A. K. (2016). Risks, Benefits, and Competitive Interference: Consumer Perceptions of Prescription Drug Versus Dietary Supplement Advertising, Journal of Current Issues \& Research in Advertising, 37(1), 59-79. https://doi.org/10.1080/10641734.2015.1119769

Schouten, A. P., Janssen, L., \& Verspaget, M. (2020). Celebrity vs. Influencer endorsements in advertising: the role of identification, credibility, and Product-Endorser fit. International Journal of Advertising, 39(2), 258-281. https://doi.org/10.1080/02650487.2019.1634898

Silchenko, K. \& Askegaard, S. (2020). Mapping moralities of food and health in marketing research $\begin{array}{llll}\text { literature. Journal of Marketing } & \text { Management, } & 36(9-10), & \text { 794-829. }\end{array}$ https://doi.org/10.1080/0267257X.2020.1791932

Soller, R. W., Rice, L. G., \& Ambrose, P. J. (2007). Disclaimers in dietary supplement print advertising: the bodybuilding category as a model case for change. Food \& Drug LJ, 62(2), 375386.

Starr, R. (2016). Should states and local governments regulate dietary supplements?. Drug Testing and Analysis, 8(3-4), 402-406. https://doi.org/10.1002/dta.1926

Stipp, H. (2018). How context can make advertising more effective. Journal of Advertising Research, 58(2), 138-145. https://doi.org/10.2501/JAR-2018-022

Teeny, J., Briñol, P., \& Petty, R. E. (2017). The elaboration likelihood model: Understanding consumer attitude change. In C. V. Jansson-Boyd \& M. J. Zawisza (Eds.), Routledge 
RLCS, Revista Latina de Comunicación Social, 79, 263-282

[Investigación] DOI: 10.4185/RLCS-2021-1511| ISSN 1138-5820| Año 2021

international handbooks. Routledge international handbook of consumer psychology (p. 390-410). Routledge/Taylor \& Francis Group.

Tran, G. A., Yazdanparast, A., \& Strutton, D. (2019). Investigating the marketing impact of consumers' connectedness to celebrity endorsers, Phychology \& Marketing, 36(10), 1-13. https://doi.org/10.1002/mar.21245

van Reijmersdal, E. A. (2011). Mixing advertising and editorial content in radio programmes: Appreciation and recall of brand placements versus commercials. International Journal of Advertising, 30(3), 425-446. https://doi.org/10.2501/IJA-30-3-425-446

Wong, V. C., Fock, H., Ho, C. K. Y. (2020). Toward a Process-Transfer Model of the Endorser Effect. Journal of Marketing Research, 57(3), 565-581. https://doi.org/10.1177/0022243720910453

\section{AUTOR/ES:}

\section{Ana García-Arranz}

Ana García-Arranz (Doctorado en la Universidad Rey Juan Carlos) es Profesora de Sociología en la Facultad de Ciencias de la Comunicación de la Universidad Rey Juan Carlos (Madrid). Sus intereses de investigación se centraron en una variedad de temas sociales como el análisis crítico del discurso, las representaciones sociales y la comunicación sanitaria. También tiene intereses en medios de comunicación y responsabilidad corporativa y periodística. Es miembro del grupo de investigación methaodos.org (grupo de metodología para la investigación social aplicada), grupo de investigación de la Universidad Rey Juan Carlos reconocido como Grupo de Alto Rendimiento por ANEP (Agencia Nacional de Evaluación y Prospectiva) en 2019.

anamaria.garcia.arranz@urjc.es

\section{Índice H: 2}

Orcid ID: https://orcid.org/0000-0002-6956-6008

Google Scholar: https://scholar.google.es/citations?user=oQyUu7kAAAAJ\&hl=es

ResearchGate: https://www.researchgate.net/profile/Ana_Garcia-Arranz

Scopus ID: $\underline{\text { https://www.scopus.com/authid/detail.uri?authorId=57217081201 }}$

\section{Salvador Perelló-Oliver}

Salvador Perelló-Oliver (Doctorado en la Universidad de Valencia) es Profesor de Metodología, Técnicas de Investigación Social y Estructura Social Contemporánea, Facultad de Ciencias de la Comunicación, Universidad Rey Juan Carlos. Sus intereses de investigación son la metodología de la investigación social, el análisis avanzado del discurso y la comunicación sanitaria. También le interesan los discursos engañosos y la manipulación de los medios y las fuentes. Ha participado en varias investigaciones financiadas y competitivas relacionadas con la regulación publicitaria y la autorregulación en la comunicación comercial relacionada con la salud. Es investigador principal de methaodos.org (grupo de metodología para la investigación social aplicada), grupo de investigación de la Universidad Rey Juan Carlos reconocido como Grupo de Alto Rendimiento por ANEP (Agencia Nacional de Evaluación y Prospectiva) en 2019.

salvador.perello@urjc.es

Índice H: 12

Orcid ID: https://orcid.org/0000-0003-2655-440X

Google Scholar: $\underline{\text { https://scholar.google.es/citations?hl=es\&user=nxDICN8AAAAJ }}$

ResearchGate: https://www.researchgate.net/search/researcher?q=salvador\%2Bperell\%25C3\%25B3 
Scopus ID: https://www.scopus.com/authid/detail.uri?authorId=55355526700

Academia.edu: https://urjc.academia.edu/SalvadorPerell\%C3\%B3Oliver

\section{Clara Muela-Molina}

Clara Muela-Molina (Doctorado en la Universidad Complutense de Madrid) es Profesora de Estrategias Creativas en Publicidad, Facultad de Ciencias de la Comunicación, Universidad Rey Juan Carlos. Sus intereses de investigación son la regulación y autorregulación publicitaria, la comunicación sanitaria y el papel de los medios de comunicación en la promoción y concienciación de la salud. También tiene intereses en la responsabilidad social de los anunciantes, los medios de comunicación y las personalidades que respaldan las comunicaciones comerciales ilícitas relacionadas con la salud. Ha participado en varias investigaciones financiadas y competitivas relacionadas con la regulación publicitaria y la autorregulación en la comunicación comercial relacionada con la salud. Es Investigadora Principal de methaodos.org (grupo de metodología para la investigación social aplicada), grupo de investigación de la Universidad Rey Juan Carlos reconocido como Grupo de Alto Rendimiento por ANEP (Agencia Nacional de Evaluación y Prospectiva) en 2019.

clara.muela@urjc.es

Índice H: 14

Orcid ID: https://orcid.org/0000-0002-1344-8731

Google Scholar: https://scholar.google.es/citations?user=1og7TbUAAAAJ\&hl=es

ResearchGate: https://www.researchgate.net/search/researcher?q=clara+muela-molina

Scopus ID: https://www.scopus.com/authid/detail.uri?authorId=55356512800

Academia.edu: https://urjc.academia.edu/ClaraMuela?subdomain=urjc 